\title{
Prevalence and Genetic Diversity of Listeria monocytogenes Isolated From Retail Pork in Wuhan, China
}

OPEN ACCESS

Edited by:

Dario De Medici,

National Institute of Health (ISS), Italy

Reviewed by:

Werner Ruppitsch,

Austrian Agency for Health and Food

Safety (AGES), Austria

Alexandre Leclercq,

Institut Pasteur, France

${ }^{*}$ Correspondence:

Mei Liu

liumei@mail.hzau.edu.cn

Specialty section:

This article was submitted to

Food Microbiology,

a section of the journal

Frontiers in Microbiology

Received: 30 October 2020

Accepted: 15 February 2021

Published: 09 March 2021

Citation:

Wang Y, Ji Q, Li S and Liu M (2021) Prevalence and Genetic Diversity of Listeria monocytogenes Isolated From Retail Pork in Wuhan, China. Front. Microbiol. 12:620482.

doi: 10.3389/fmicb.2021.620482

\author{
Yiqian Wang ${ }^{1,2}$, Qiang Ji', Shaowen $\mathrm{Li}^{1}$ and Mei Liu' ${ }^{1 *}$ \\ ${ }^{1}$ College of Veterinary Medicine, Huazhong Agricultural University, Wuhan, China, ${ }^{2}$ College of Life Science and Technology, \\ Huazhong Agricultural University, Wuhan, China
}

Listeria monocytogenes is a ubiquitous bacteria and causative agent of zoonotic listeriosis with high mortality. The consumption of contaminated animal-derived foods has been linked with both epidemic and sporadic listeriosis. In this work, a total of 64 L. monocytogenes isolates from 259 pork samples sold in 11 supermarket chains were identified and characterized by comparative whole-genome analysis. All isolates were delineated into eight clonal complexes (CCs), namely CC2, CC8, CC9, CC11, CC155, CC121, CC204, and CC619, spanning two lineages (I and II) and carrying 35 antibiotic-resistant genes (fos $X$, Inu, mprF, tetM, and $d h f R$ ). It is noted that Listeria pathogenicity island (LIPI)-1, LIPI-3, and LIPI-4 were distributed in all ST619 isolates from two supermarket chains that were closely related with clinical isolates ( $<40 \mathrm{SNP}$ ). Some of the isolates from different supermarket chains with 0 SNP difference indicated a common pork supply source. Notably, $57.81 \%$ of the strains carried types IB, IIA, or IIIB CRISPR-Cas system, CC121 isolates carried both types IB and IIA CRISPR-Cas systems, Cas proteins of CC155 isolates located between two CRISPR loci, each CC has unique organization of Cas proteins as well as CRISPR loci. CRISPR-Cas systembased subtyping improved discrimination of pork-derived $L$. monocytogenes isolates. Comparisons at the genome level contributed to understand the genetic diversities and variations among the isolates and provided insights into the genetic makeup and relatedness of these pathogens.

Keywords: Listeria monocytogenes, genetic diversity, pathogenic island, antibiotic-resistant genes, ST619, cgMLST, SNP, CRISPR-Cas system

\section{INTRODUCTION}

Listeria monocytogenes is a Gram-positive, facultative intracellular pathogen that is ubiquitously distributed in the environment (Smith et al., 2019). A wide variety of animal species can be infected by L. monocytogenes, including mammals, birds, fish and crustaceans (OIE terrestrial manual 2018. Chapter 3.9.6 - L. monocytogenes). Pigs rarely develop listeriosis when exposed to L. monocytogenes contaminated feed (OIE terrestrial manual 2018. Chapter 3.9.6 - L. monocytogenes), while they may become carriers of L. monocytogenes, and the pathogen is transmissible to humans through the pig and pork production chain to cause human listeriosis (Félix et al., 2018). Listeriosis outbreaks are reported from time to time worldwide. In South Africa, an outbreak claimed the lives of 193 patients caused by Listeria contaminated pork sausage in 2017 (Thomas et al., 2020). In France, an outbreak (32 cases, 10 deaths) occurred because of $L$. monocytogenes contamination in pork product in 
1999-2000 (Matle et al., 2020). In United States, CDC reported an outbreak of Listeria infection linked to pork products in $2018^{1}$. The contamination and transmission of L. monocytogenes along pork production chain poses serious threats to public health and food safety, it has become a concern for countries around the world.

Understanding the prevalence of L. monocytogenes in pork can provide fundamental data to evaluate the risk of Listeria infecting human. The investigations of L. monocytogenes contamination in pork have been carried out in most countries. In the European Union in 2018, L. monocytogenes in pig meat was detected in $1.3 \%$ of the 24,814 units tested, and in $0.8 \%$ ( 11 out of 1,365 ) of the tested samples at retail (EFSA and ECDC, 2019). In South Africa, the occurrence of L. monocytogenes was higher in pork $(13.3 \% ; 18 / 135)$ in 2014-2016 (Matle et al., 2019). In Mexico, L. monocytogenes was present in 16/79 (20.3\%) pork samples collected from two slaughterhouses from 2014 to 2015 in southern Sonora (Figueroa-López et al., 2019). In China, L. monocytogenes contaminations in retail raw pork (11.68-30\%), chilled pork (11-55\%), and RTE pork (4.61-13.5\%) have been reported in recent years (Yin et al., 2015; Li et al., 2018; Chen et al., 2019; Zhang et al., 2019b). Pork is the most frequently consumed meat in China, the main consumption habit is to take cooked pork. Whereas, with the change of lifestyle, the frequency of consumption RTE food is increasing, the contamination in RTE pork (4.61-13.5\%) suggested that cross-contamination in the process of cooked food increased the infection risk to human. However, the system of surveillance is not clearly described at food and clinical levels.

Listeria monocytogenes is a highly heterogeneous species; its population structure is divided into 14 serotypes (Yin et al., 2019) and four phylogenetic lineages (I, II, III, and IV) (Orsi et al., 2011) that have been classified into multiple clonal complexes (CCs) on the basis of multilocus sequence typing (MLST) (Ragon et al., 2008). In the western countries, CCs (1-6) of lineage I are the major types responsible for human epidemics. Further, CC8, CC9, CC121, and CC155 of lineage II are the main causative CCs of human listeriosis; however, their frequency of being reported is lower than that of lineage I isolates, which mainly cause sporadic listeriosis (Chenal-Francisque et al., 2011; Chen et al., 2016; Maury et al., 2016). In China, listeriosis epidemics are different from those in other countries; outbreaks are rarely reported, and sporadic cases are more common, which can be attributed to certain relationships among the predominant clones CC87, CC8, and CC9 in food (Li et al., 2019; Yin et al., 2019; Zhang et al., 2019a). Particularly, the previous studies reported the circulation of typical L. monocytogenes CC87 clone in food and clinical disease (Wang et al., 2019; Yin et al., 2020). L. monocytogenes is a relatively conservative and constantly varying species. Dynamic epidemic investigations are particularly necessary to understand the genetic diversities and structures of related virulent genes, antibiotic-resistant genes, and horizontal moving elements as well as CRISPR-Cas system.

Wuhan city in Hubei province has 14 million residents who are mainly of the Han ethnicity, and pork meat is the most often

${ }^{1}$ https://www.cdc.gov/listeria/outbreaks/index.html consumed animal product in their diet. Although people have gradually been paying more attention to food safety, epidemic investigation data on pork meat in Wuhan city is still limited. In this study, the L. monocytogenes contamination status of pork meat in 53 supermarkets belonging to 11 supermarket chains was investigated. The genomic analyses for genetic diversity, virulence, antibiotic resistance, and CRISPR-Cas systems of L. monocytogenes isolates were performed. Our research is expected to be beneficial for understanding the prevalence and characterization of Listeria contamination in retail pork in China, which is necessary for effectively solving problems related to animal-derived foods and ensuring safety from farm to table.

\section{MATERIALS AND METHODS}

\section{Isolation}

A total of 259 raw pork meat samples were collected from 53 supermarkets in three districts in Wuhan city between January and August in 2019, including Wuchang $(n=62)$, Hankou ( $n=75)$, and Hanyang $(n=122)$ (Figure 1). The details of L. monocytogenes strains isolated from pork in Wuhan can be found in Table 1. All the samples were collected and transported to the laboratory at $4^{\circ} \mathrm{C} \pm 2^{\circ} \mathrm{C}$ on the same day. Twenty-five-gram pork samples were selectively enriched by UVM modified Listeria enrichment broth (Becton, Dickinson and Company, Sparks, MD, United States) at $30^{\circ} \mathrm{C}$ for $24 \mathrm{~h}$. Then, $100 \mu \mathrm{L}$ of the UVM culture was added and selectively enriched in $10 \mathrm{~mL}$ FB medium (Becton, Dickinson and Company, Sparks, MD, United States) at $37^{\circ} \mathrm{C}$. The FB culture was then inoculated and spread on CHROMagar ${ }^{\mathrm{TM}}$ plates (Shanghai KeMaJia Biological Technology Co., Ltd., Shanghai, China) at $37^{\circ} \mathrm{C}$. The colonies with blue/green sheens and opaque halos on CHROMagar ${ }^{\mathrm{TM}}$ plates were inoculated on Bacto ${ }^{\mathrm{TM}}$ brain and heart infusion medium (BHI, Becton, Dickinson and Company, Sparks, MD, United States) plates. Typical colonies on BHI plates were identified according to the method reported by Bubert et al. (1999).

\section{Serotype Identification}

The multiplex PCR method was used to detect the PCR serogroups of all isolates according to Doumith et al. (2004). Then, based on PCR serogrouping, antigen serum agglutination and slide agglutination were performed using Listeria $\mathrm{O}$ and $\mathrm{H}$ antisera following manufacturer's instructions (Denka Seiken Co. Ltd., Tokyo, Japan) to determine the serotypes of isolates.

\section{Genome Sequencing}

The genomes of the isolates were extracted using the TaKaRa MiniBEST Bacteria Genomic DNA Extraction Kit Ver.3.0 (TAKARA, Dalian, China) according to manufacturer's instructions. Genome sequencing was carried out by the Shanghai Majorbio company. DNA libraries harboring 400 bp DNA fragments were prepared using the NEXTflex ${ }^{\text {TM }}$ Rapid DNA-Seq following manufacturer's instructions and pair-end sequenced $(2 \times 150 \mathrm{bp})$ on the HiSeq $\times 10$ system (Illumina, San Diego, CA, United States). The quality of the sequence 




FIGURE 1 | Distribution of supermarket chains in Wuhan. The names of 11 supermarket chains distributed in Hanyang, Hankou and Wuchang were labeled on the

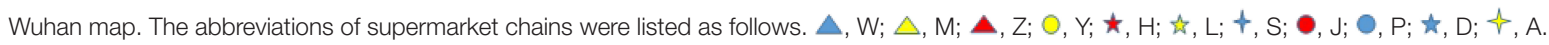

criteria was Q20 $\geq 90 \%$, the depth of sequencing was 100 -fold of the genome coverage. The original image data is transferred into sequence data via base calling, which is defined as raw reads and saved as FASTQ file. Quality information by Fastp software V0.20.1 ${ }^{2}$ was applied for quality trimming, by which the low-quality data was removed to form clean data. The assembly of the clean reads was performed using SOAPdenovo $2^{3}$. The sequence reads for 64 isolates are deposited and publicly available under the bioproject accession number PRJEB41790. Publicly available genome sequences of 20 clinical isolates were referred to compare the evolutionary relationship (Supplementary Table 2).

\section{Comparative Genome Analysis MLST and Core Genome MLST (cgMLST)}

Sequence types (STs) of the isolates were determined by multilocus sequence typing (MLST), as previously described (Salcedo et al., 2003). The clonal relationships of isolates based on MLST were obtained based on the similarities of the allelic profiles of the seven housekeeping genes to assign the ST as established by the Institut Pasteur MLST ${ }^{4}$ and MLST database ${ }^{5}$ (Ragon et al., 2008).

The cgMLST analysis was carried out using cgMLSTFinder $1.1^{6}$. The 1,748 core genome multilocus sequence typing (cgMLST) loci used in this study were obtained from BIGSdbLm platform ${ }^{7}$ (Moura et al., 2016). Phylogenetic analysis of 64 isolates based on 1,748 cgMLST loci was performed using cgmlstfinder.py contained in the cgMLSTFinder service (Clausen et al., 2018). iTOL was used to make the phylogenetic tree visual (Letunic and Bork, 2007).

\footnotetext{
${ }^{2}$ https://github.com/OpenGene/fastp

${ }^{3}$ https://github.com/aquaskyline/SOAPdenovo2

${ }^{4} \mathrm{http}: / /$ www.pasteur.fr/mlst

${ }^{5}$ https://cge.cbs.dtu.dk/services/MLST/

${ }^{6} \mathrm{https} / / /$ cge.cbs.dtu.dk/services/cgMLSTFinder/

${ }^{7}$ http://bigsdb.pasteur.fr/listeria
}

\section{Virulent Gene Typing}

The assembled genomes were uploaded to the online analysis software virulence finder 2.0 created by the Center for Genomic Epidemiology $\left(\mathrm{CGE}^{8}\right)$. Pathogenicity island (LIPI)-1, -3, and -4 as well as genes associated with adhesion, invasion, survival, etc., were analyzed with the Database for Virulence Factors of Pathogenic Bacteria (VFDB) (Chen et al., 2005) and ResFinder (Zankari et al., 2012). Virulence genes were considered present when identity was $>90 \%$ and the coverage was $>80 \%$. Genes belonging to LIPI- $1,-3$, and -4 were detected using BLASTN software. The phylogenetic tree was determined with BioNumerics v7.5 based on the spacer arrangements of virulent genes for each strain.

\section{Antibiotic-Resistant Gene Detection}

Antibiotic-resistant genes in the isolates were analyzed using the online platform ${ }^{9}$ (Alcock et al., 2020) with the selection criterion as "Perfect and strict hits only," Nudge $\geq 95 \%$ identity Loose hits to Strict (exclude nudge). The sequences of antibiotic genes and annotations were downloaded, and a maximum likelihood phylogenetic tree was constructed based on the antibiotic profiles using MEGA X software.

\section{SNP Typing}

Genome sequencing data were obtained by quality filtration and adaptor clearance by fastp. The reads were aligned to reference EGDe using BWA software (Li and Durbin, 2009) and converted to a BAM file. The strains and single nucleotide variations (SNVs) were analyzed with GATK v3.5 (HaplotypeCaller) with the de novo algorithm and obtained using the VCF file containing the SNV mutation sites (McKenna et al., 2010). Core single-nucleotide polymorphism (SNP) alignments were used to construct the maximum likelihood phylogenetic tree using SNPhylo pipeline with 1000 bootstraps.

\footnotetext{
${ }^{8}$ https://cge.cbs.dtu.dk/services/VirulenceFinder/

${ }^{9}$ https://card.mcmaster.ca/analyze/rgi
} 
TABLE 1 | Details of L. monocytogenes strains isolated from pork in Wuhan.

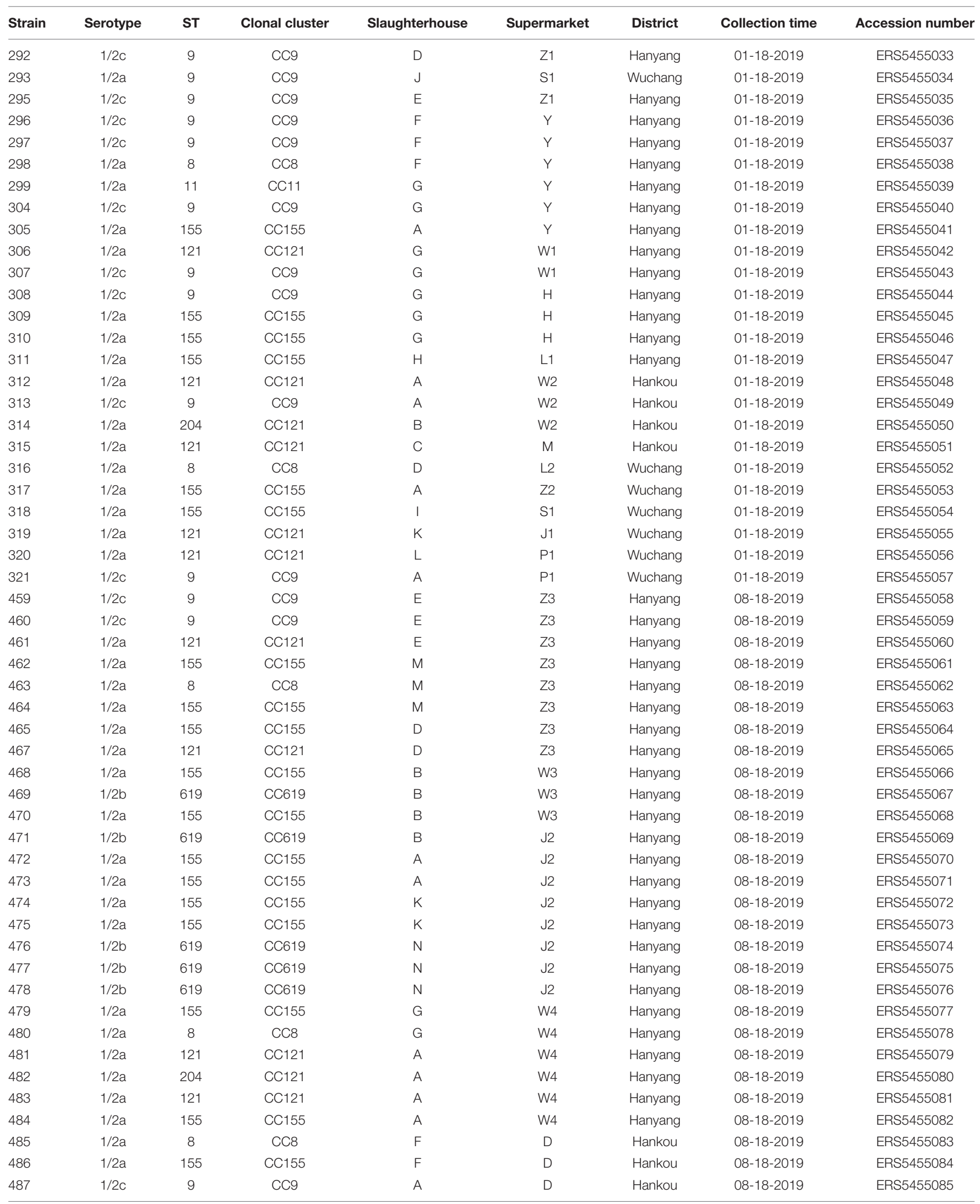


TABLE 1 | Continued

\begin{tabular}{|c|c|c|c|c|c|c|c|c|}
\hline Strain & Serotype & ST & Clonal cluster & Slaughterhouse & Supermarket & District & Collection time & Accession number \\
\hline 488 & $1 / 2 \mathrm{a}$ & 155 & CC155 & I & D & Hankou & 08-18-2019 & ERS5455086 \\
\hline 489 & $1 / 2 b$ & 2 & CC2 & I & $D$ & Hankou & 08-18-2019 & ERS5455087 \\
\hline 490 & $1 / 2 a$ & 155 & CC155 & $\mathrm{B}$ & W5 & Hanyang & 08-18-2019 & ERS5455088 \\
\hline 491 & $1 / 2 \mathrm{a}$ & 204 & CC121 & $\mathrm{B}$ & W5 & Hanyang & 08-18-2019 & ERS5455089 \\
\hline 492 & $1 / 2 \mathrm{a}$ & 204 & CC121 & A & W5 & Hanyang & 08-18-2019 & ERS5455090 \\
\hline 493 & $1 / 2 a$ & 155 & CC155 & A & W5 & Hanyang & 08-18-2019 & ERS5455091 \\
\hline 494 & $1 / 2 \mathrm{a}$ & 204 & CC121 & $J$ & W5 & Hanyang & 08-18-2019 & ERS5455092 \\
\hline 495 & $1 / 2 \mathrm{a}$ & 204 & CC121 & $J$ & W5 & Hanyang & 08-18-2019 & ERS5455093 \\
\hline 496 & $1 / 2 a$ & 204 & CC121 & G & W5 & Hanyang & 08-18-2019 & ERS5455094 \\
\hline 497 & $1 / 2 a$ & 155 & CC155 & G & W5 & Hanyang & 08-18-2019 & ERS5455095 \\
\hline
\end{tabular}

\section{CRISPR Typing}

CRISPR spacers and repeats were identified using CRISPRFinder $^{10}$ (Grissa et al., 2007). The similarity of each spacer to the sequences deposited in GenBank was analyzed by BLASTN using an e-value cutoff of 0.1. The features of CRISPR in each isolate were input to the binary table, and the maximum likelihood phylogenetic tree was inferred using BioNumerics v7.5 according to the spacer arrangements in each strain.

\section{RESULTS}

\section{Abundant Diversity of Isolates From Supermarket Chains}

A total of $64 \mathrm{~L}$. monocytogenes strains were isolated from 259 pork samples $(24.71 \%)$ in 53 supermarkets belonging to 11 supermarket chains, which were supplied from 14 slaughterhouses (Table 1, Supplementary Figures 1A,B, and Supplementary Table 1). The isolation rate of samples collected from Hanyang $(39.34 \% ; 48 / 122)$ was obviously higher than those from Wuchang (11.29\%; 7/62) and Hankou (12.00\%; 9/75) (Supplementary Figure 1A). Additionally, the isolation rates of samples collected in August were higher than those for samples collected in January (42.00\% vs. $15.38 \%$ ). For example, these values for $\mathrm{W}$ supermarkets were $48.75 \%$ vs. $13.51 \%, \mathrm{Z}$ supermarkets were $40.00 \%$ vs. $13.64 \%$, and J supermarkets were $33.30 \%$ vs. $4.76 \%$ (Table 1). The isolation rate of samples from $\mathrm{W}$ supermarkets was higher than other supermarkets (Supplementary Figure 1B), thus it suggested that the pork sold in this supermarket chain was more contaminated. The isolation rates of W supermarkets were highest for either samples collected in January or August (Table 1). 64 isolates comprised serotypes $1 / 2 \mathrm{a}, 1 / 2 \mathrm{~b}$, and $1 / 2 \mathrm{c}$ (Supplementary Figure 1C) belonging to the eight STs $(2,8,9,11,121,155,204$, and 619), the predominant STs were ST155, ST121, and ST9 (Figure 2 and Supplementary Figure 1D).

The high differentiation characterizations of the cgMLST displayed on the neighbor-joining tree (Figure 3) were based on 1,748 core genes. All the obtained isolates were classified into eight CCs, CC8, CC9, CC121, CC155, CC204, CC619, CC2,

\footnotetext{
${ }^{10} \mathrm{https}: / /$ crisprcas.i2bc.paris-saclay.fr/CrisprCasFinder/Index
}

and CC11. Strikingly, two pairs of CCs, CC2 and CC619 or CC9 and CC204 presented closely phylogenetic relationship. Additionally, three groups in the $\mathrm{CC} 155$ cluster and two groups in either CC121 or CC9 cluster clearly demonstrated powerful differentiation characterizations for cgMLST. Interestingly, CC619 strains isolated from supermarket J2 and W3 had closely relationship, which were supplied by the same slaughterhouse B. Isolates $\mathrm{Lm} 485$ and $\mathrm{Lm} 298$ belonging to CC8 were from supermarket D and Y, respectively, which were supplied from the same slaughterhouse F. Notably, some strains isolated from contaminated pork in different supermarket chains which were supplied from different slaughterhouses were clustered to the same branch. cgMLST analysis can reflect the genetic diversity and relationship, has high discriminatory and traceability, and is a powerful tool to improve surveillance.

\section{Resistance to Multiple Antibiotic Agents}

The phylogenetic tree was constructed with sequences of the antibiotic-resistant genes (Figure 4). The entire 64 isolates were grouped into two branches: all $1 / 2 \mathrm{~b}$ strains $(\mathrm{ST} 2,619)$ belonged to clade B, and $1 / 2 \mathrm{a}$ and $1 / 2 \mathrm{c}$ strains (ST 8, 9, 11, 121, 155, and 204) were part of clade A. The strains belonging to clade A were further classified into two branches comprising different ST isolates. Interestingly, each ST strain was clustered within the same group and harbored the same antibiotic-resistant genes (Figure 4). Importantly, fosfomycin resistant genes fos $X$, lincosamide resistant gene $\ln u$, and multiple peptide resistant gene $m p r F$, were present in all the isolates. Additionally, ST204, ST121, and ST9 (except isolate Lm483) strains harbored another resistance gene $m f s$. The ST155 strains were divided into two groups, except for the three antibiotic-resistant genes mentioned above, one group carried the tetracycline-resistance gene (tet $M)$, and the other carried tet $M$ and gene-encoded dihydrofolate reductase ( $d h f R$ ), our study was the first report on ST155 carrying $d h f R$. Isolates presenting high resistances to fosfomycin have been reported in various countries (Harakeh et al., 2009; Hasegawa et al., 2013). Notably, Scortti et al. $(2006,2018)$ found that most L. monocytogenes strains resisted to fosfomycin in vitro-in vivo paradox, an epistatic interaction between virulence and resistance genes controlled bacterial susceptibility to fosfomycin in vivo. The antimicrobial resistance gene $m p r F$ that was exclusively present in $\mathrm{CC} 1, \mathrm{CC} 8$, and $\mathrm{CC} 121$ was reported in clinical and 


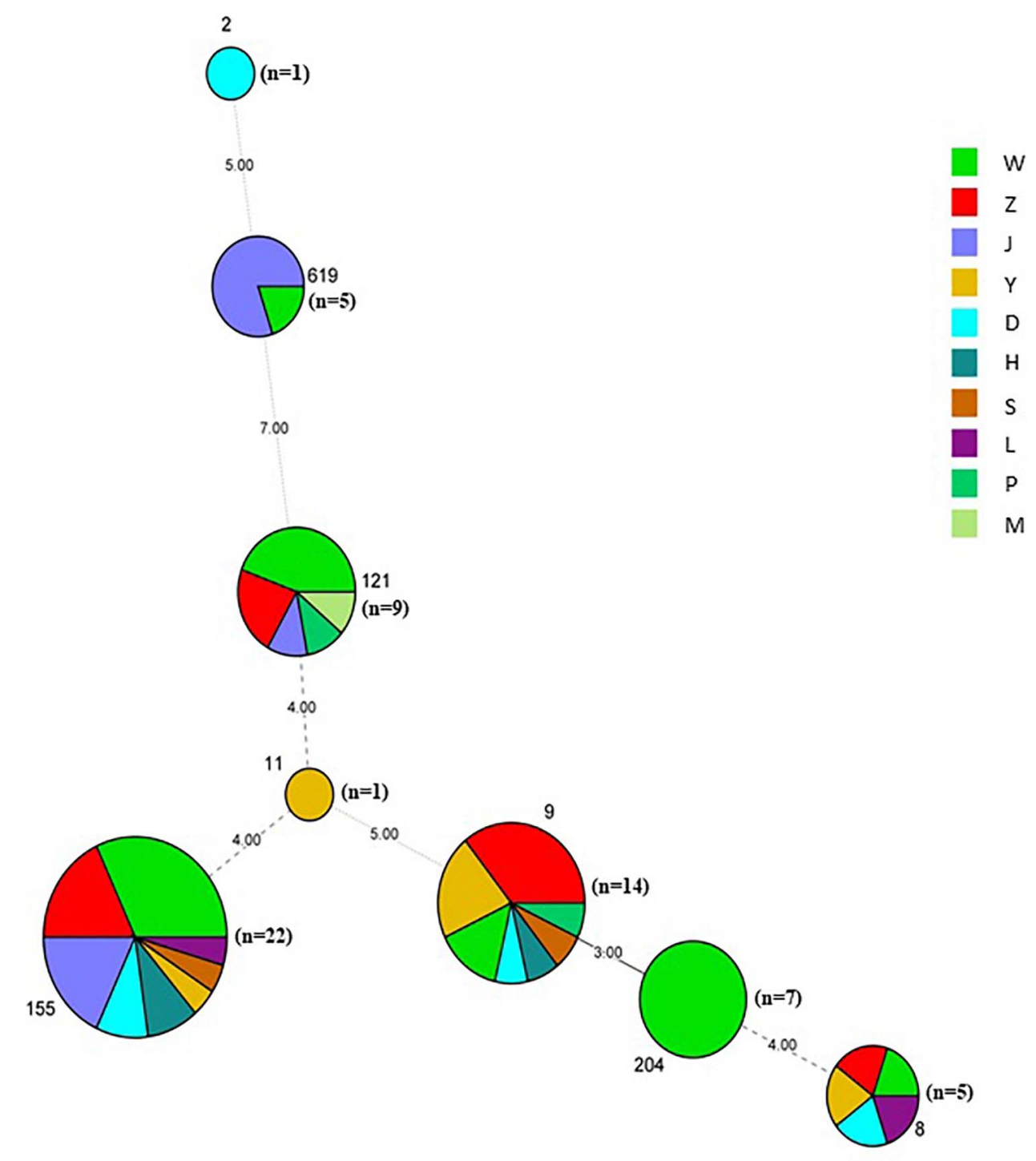

FIGURE 2 | Minimum spanning tree of MLST data for 64 L. monocytogenes isolates. Each circle represents one ST, the size of which is related to the number of strains within the ST. The branch numbers account for the number of allele differences between the connected STs. The colors of the circles represent the supermarket chains as indicated. The circle size represents number of isolates, ST2 $(n=1)$, ST8 $(n=5), \operatorname{ST9}(n=14)$, ST121 $(n=9)$, ST619 $(n=5)$, ST11 $(n=1)$, ST155 $(n=22)$, and ST204 $(n=7)$.

food isolates (Zuber et al., 2019). Moreno et al. (2014) reported that isolates from slaughterhouse environments, pork and human infections were found to harbor $m p r F$. Considering the intricate characteristics of intrinsic and acquired antibiotic resistance, it is significant to determine the antibiotic phenotype and mechanism of these isolates.

\section{Presence of LIPI-1, LIPI-3, and LIPI-4 in Listeria Isolates}

In this study, a total of $64 \mathrm{~L}$. monocytogenes strains isolated from 11 supermarket chains spanned lineages I and II. The proportions of these isolates within lineages I and II were 90.6 and 9.4\%, respectively. LIPI-1 was found in all the isolates, and none of the isolates harbored LIPI-2; LIPI-3 was found in all ST619 and ST2 isolates belonging to lineage II, and LIPI-4 was only distributed in ST619 isolates (Figure 5). Unusually, the ST619 isolates carried three Listeria pathogenic islands (LIPI-1, -3 , and -4).

To investigate the virulence potentials of the 64 isolates, an in silico detection of 91 virulent and virulence-related genes was carried out, main virulent genes in each isolate were presented in Figure 5. Interestingly, either lisR or lisK was absent in the eight STs, inlL was absent in all isolates, vip gene was not detected in the ST2, ST8, ST204 and ST619 strains. All ST8, ST11 and 


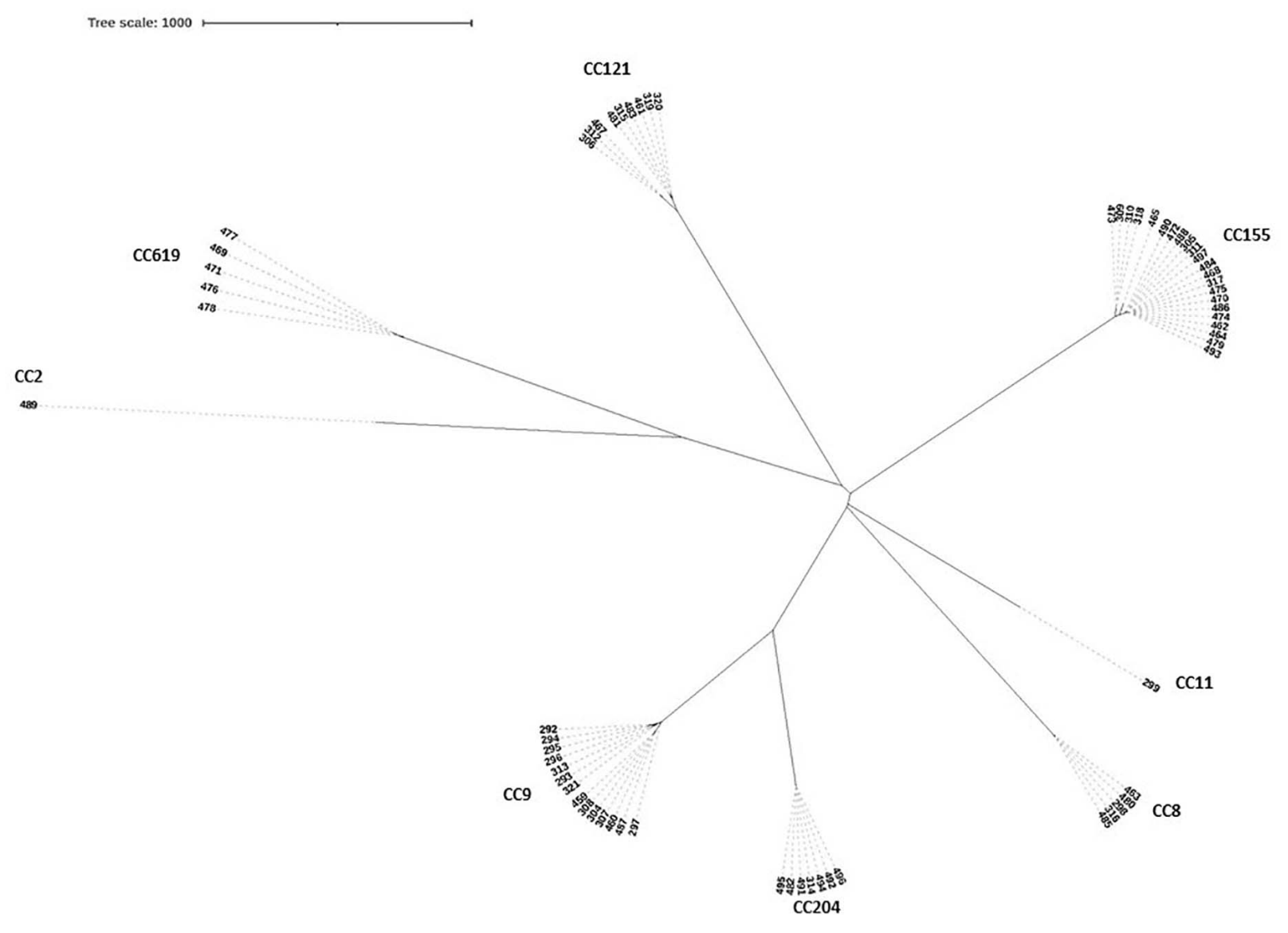

FIGURE 3 | Neighbor-joining tree of $L$. monocytogenes isolates as determined by cgMLST based on the 1,748 core genes. The neighbor joining tree shows the relatedness of the $64 \mathrm{~L}$. monocytogenes isolates, each cluster was corresponding to specific CC, the name of per CC was labeled on related cluster.

ST155 strains carried inlF gene. inlA encoded truncated InlA presented in 9 ST121 isolates and 3 ST9 isolates. Parts of the ST strains did not contain ami and aut. Virulent and virulenceassociated genes are widely distributed in 64 isolates, particularly the ST619 strains, indicating high virulence features and potential high risk to humans.

\section{Phylogenetic Relationship of Isolates From Multiple Supply Chains}

The SNP of each isolate was obtained in comparison with the EGDe strain (Figure 6 and Supplementary Table 3), 8 clusters comprising 8 CCs were consistent with the phylogenetic tree using cgMLST. A part of the isolates from different supermarket chains showed high genetic similarity (0-50 SNP differences). For example, isolates Lm305, Lm317, and Lm484 were isolated from the supermarket chain $\mathrm{Y}, \mathrm{Z}$, and $\mathrm{W}$, respectively, however, showed 4 SNP differences between Lm317 and Lm484, and 45 SNP differences between Lm317 and Lm305. Because the pork meats of these 3 supermarket chains were supplied by the same slaughterhouse A, it suggested that the contamination of pork was possibly sourced from the same production chain. Additionally, the isolates were from different supermarket chains and collection times, but the SNP differences were less than two. For example, despite the temporal (half year gap) and geographical differences among the isolates, Lm459, Lm304, Lm487, and Lm307 indicated the persistence of specific L. monocytogenes in the pork production chain. 12 strains comprising 5 STs were isolated in pork meat from supermarket chain J, Z, and W, which were supplied by the same slaughterhouse $\mathrm{E}$; the pigs transported to the slaughterhouse E were from four different pig farms (HP, SP, GS3, and YJ). Therefore, various diversity of the strains was possibly due to the intricate supply chain. Importantly, specific ST isolates such as ST8 showed high genetic similarity ( $<21$ SNP differences) to clinical isolates Lm 1823 and SHL004 (Supplementary Table 2). This represented a public health concern because of their potential to transfer to humans via the food chain.

\section{CRISPR-Cas System-Based Subtyping Contributing to Improve the Discrimination}

Analysis of the CRISPR-Cas system of isolates revealed that the system was distributed in most of the isolates (37/64, 57.81\%). Based on the number and diversity of the CRISPR-Cas system, 

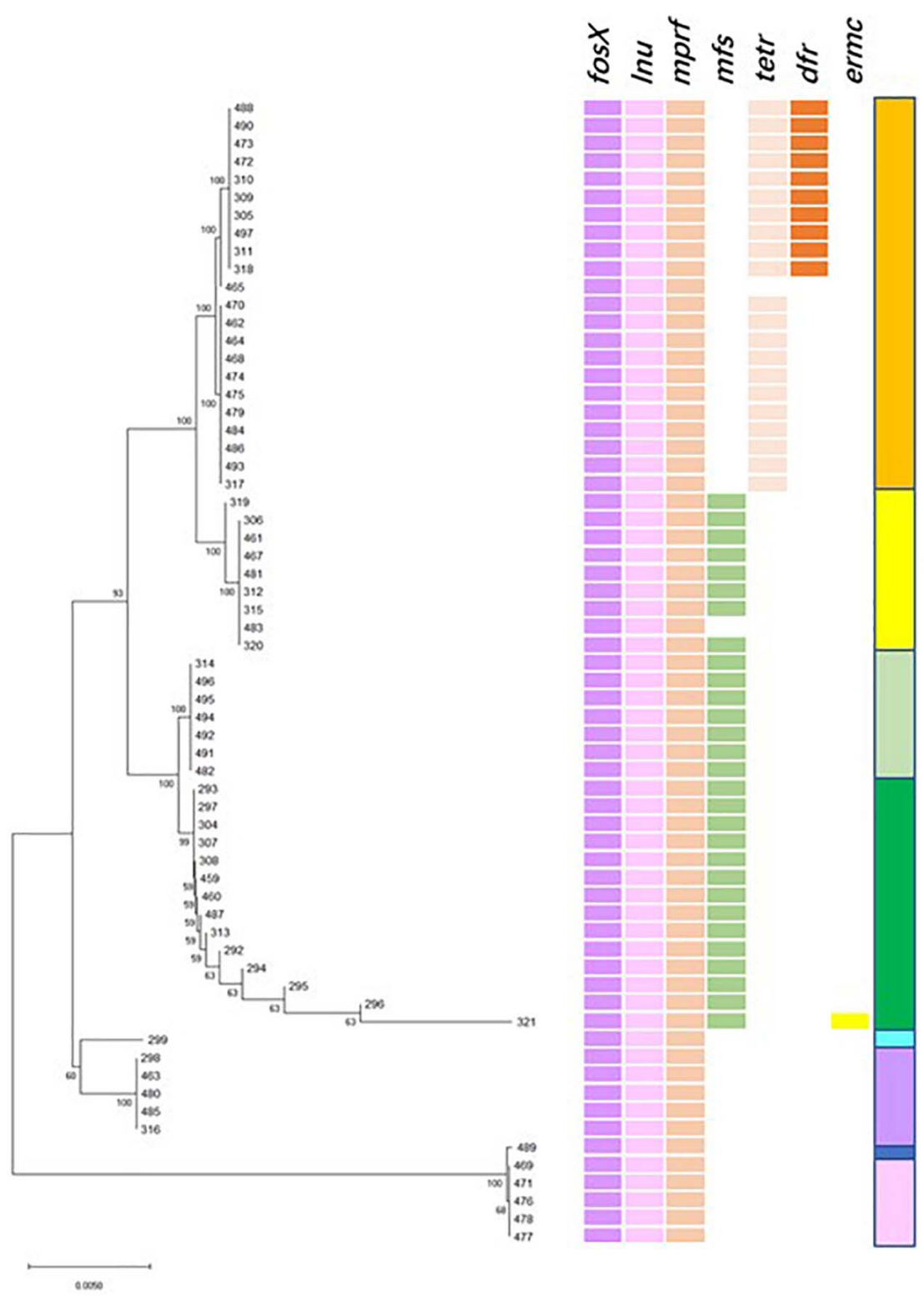

ST155

ST121

ST204

ST9

ST11

ST8

ST2

ST619

FIGURE 4 | Resistance genes profile. (Left) Maximum likelihood phylogenetic tree based on the antibiotic-resistant genes. (Right) Patterns of antibiotic-resistant gene presence (colored line) or absence (white). The right column corresponds to the sequence type.

these isolates were divided into two classes (classes 1 and 2) and three types (types IB, IIA, and IIIB) (Figure 7 and Table 2). Among 8 STs, only ST121, ST155, and ST619 isolates harbored complete CRISPR-Cas system. Interestingly, all of the ST121 strains carried both types IB and IIA CRISPR-Cas systems (Figure 7 and Table 2), and CRISPR loci of ST155 isolates existed in both upstream and downstream of Cas proteins. Other STs only carried Cas10 protein or one to four Cas3 proteins, particularly of carrying limited spacers (between 0 and 10) (Table 2). The ST9 isolates carried either Cas10 or Cas3 proteins (Table 2). As mentioned above, the genotypes of the CRISPR-Cas system were observed to be correlated with the STs.
It is worth noting that there was variant organization of CRISPRs in the same ST (Table 2 and Supplementary Figure 2), for example, either of type IIA CRISPR-Cas system of ST121 isolates or types IB CRISPR-Cas system of ST155 isolates comprised 7 kinds of CRISPRs. The diversity of the CRISPR-Cas system indicates the role of various functions in the corresponding strains, which are possibly related to different STs encountered in distinct environments. Interestingly, 13 pairs of the isolates (0 SNP difference) were indistinguishable by SNP subtyping, but these isolates could be differentiated adequately by CRISPR-Cas subtyping. For example, three 0-SNP-difference strains isolated from the same supermarket chain were found to harbor various CRISPR spacers. In brief, 


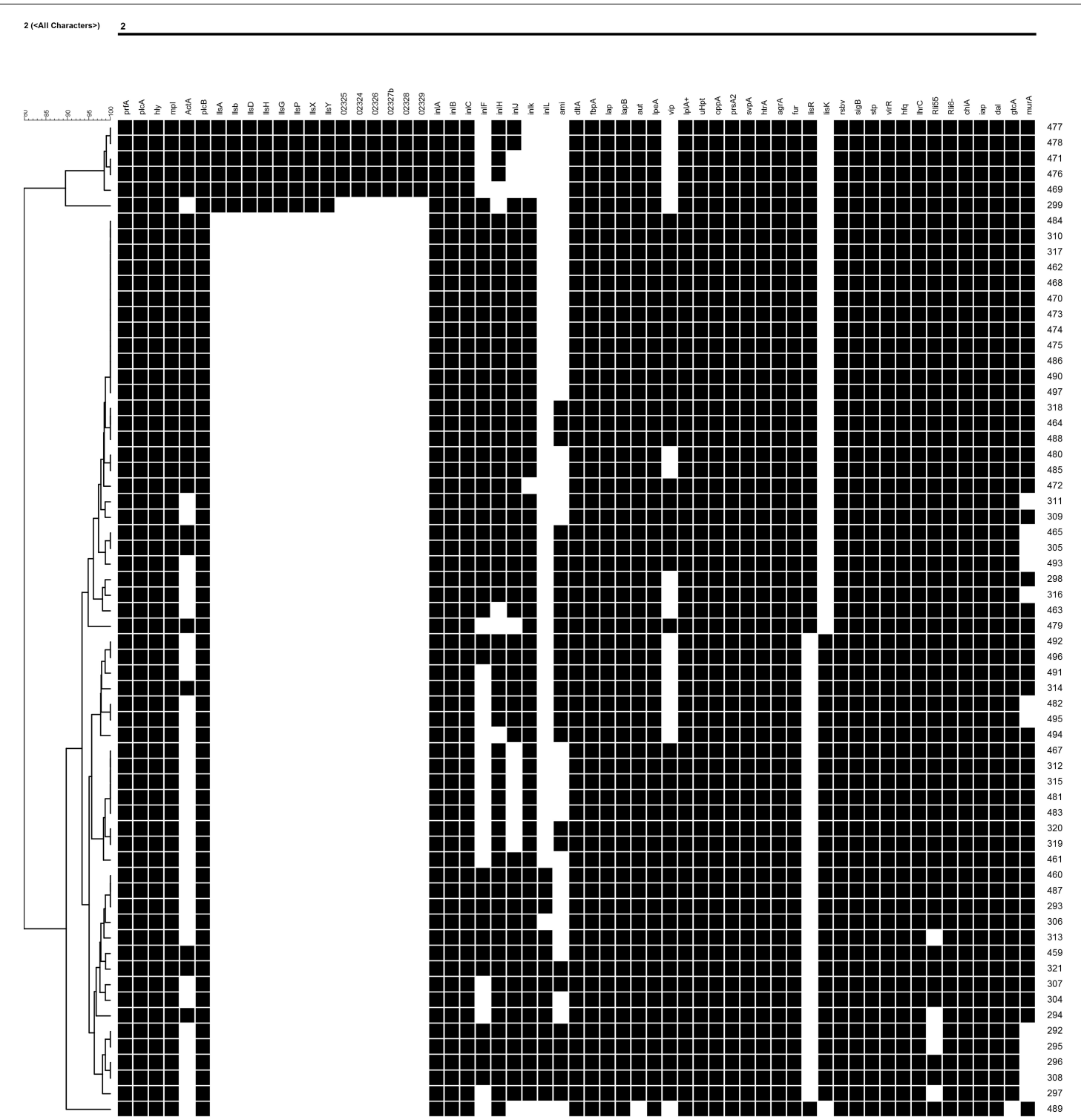

FIGURE 5 | Virulence profiles across the phylogeny of the L. monocytogenes isolates. (Left) Phylogenetic tree of all isolates determined by 59 virulent and virulence-related genes, part of genes belong. The phylogenic relationship among different STs is shown by the phylogenic tree. (Right) Pattern of gene presence (colored line) or absence (white). The presence/absence gene matrix represents, from left to right, genes located in the pathogenicity islands LIPI-1 (prfA, plcA, $h l y$, mpl, actA, and plcB), LIPI-3 (IISAGHXBYDP) and LIPI-4 (02325, 02324, 02326, 02328, and 02329), genes coding for internalins (in/ABCFHJKL) and other genes involved in adherence (ami, dltA, fbpA, lap, and lapB), invasion (aut, IpeA, and vip), intracellular survival (hpt, IplA1, oppA, prs $A 2$, purQ, and svpA), regulation of transcription and translation (agrA, fur, lisR, lisK, sbV, sigB, stp, virR, and hfq), small non-coding RNA (IhrC, Rli55, and Rli6), cell wall synthesize (chiA, iap, dal, gtcA, and murA).

these results verify that the organization of the CRISPR-Cas system exhibits genetic diversity of isolates, thereby reflecting the higher discrimination ability.

\section{DISCUSSION}

Listeria monocytogenes is a severe foodborne pathogen and is a significant threat to public health. Animal-derived Listeria-contaminated food is generally understood to be the main cause of listeriosis outbreaks (de Valk et al., 2001; Choi et al., 2014), this pathogen with the evidence of zoonotic transmission causes global economic and health burdens (Halbedel et al., 2020; Thomas et al., 2020). Therefore, improving the detection of bacterial contamination of pork, beef and poultry is an efficient measure to prevent human listeriosis.

Genome sequencing has been gradually used to reveal the detailed characteristics of typing, virulent, and antibioticresistant genes and has become a useful investigation tool 


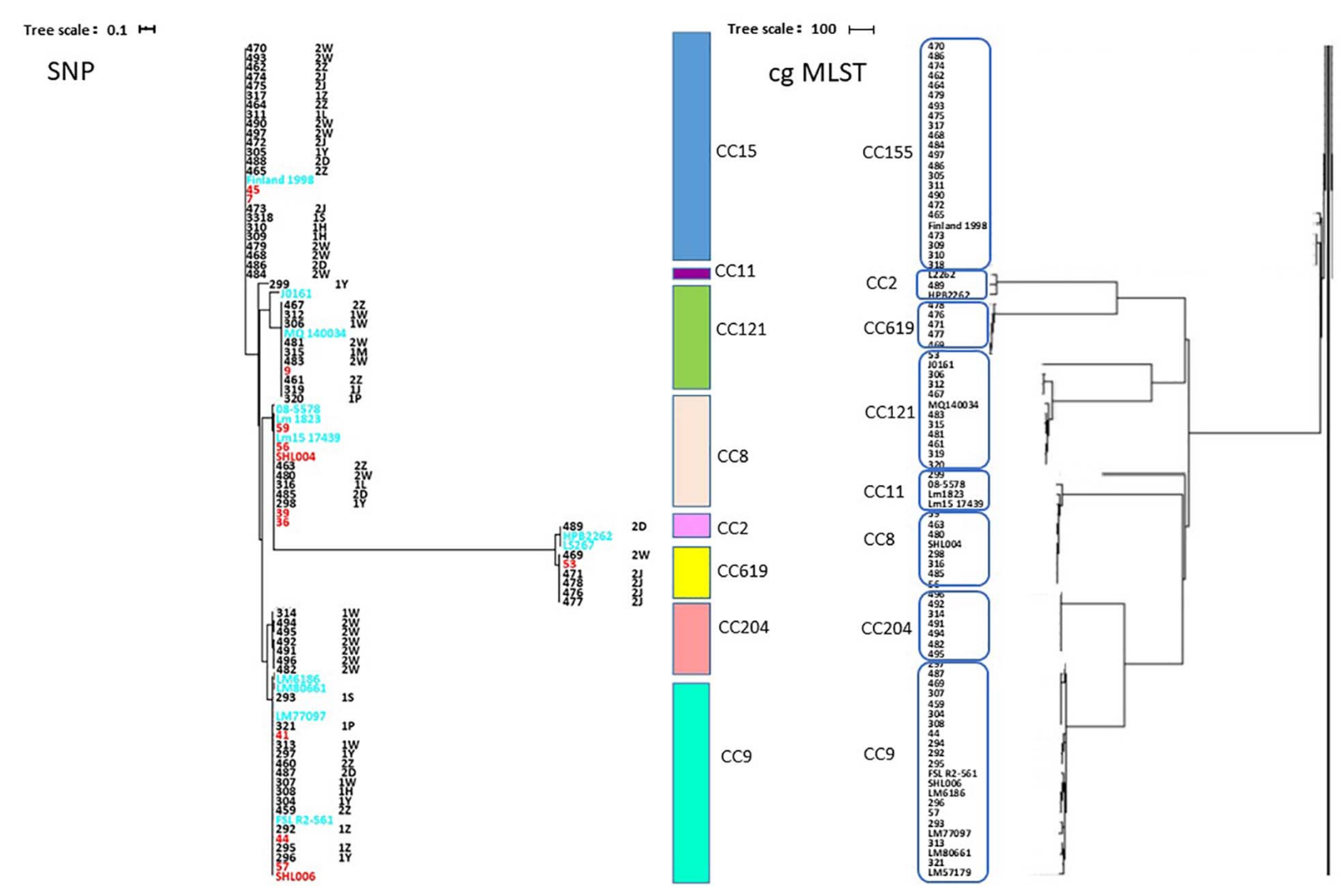

FIGURE 6 | Phylogenetic trees of 64 isolates and 20 clinical isolates constructed from SNPs and cgMLST. (Left) SNPs were identified by the SNPhylo pipeline using EDGe as the reference. Strains labeled with light blue color are foreign clinical isolates. Strains labeled with red color are clinical isolates from China. The right column shows the CCs corresponding to the branches of the phylogenetic tree. The words after the names of the isolates are the isolation times and supermarket chains.

"1" represents the strains isolated in January 2019. "2" represents the strains isolated in August 2019. The uppercase letters are the abbreviations of the supermarket chains. (Right) Neighbor-joining tree of $L$. monocytogenes strains as determined by cgMLST based on the 1,748 core genes. The neighbor-joining tree shows the relatedness of the $64 \mathrm{~L}$. monocytogenes isolates and 20 clinical isolates, each blue circle represents one specific CC.

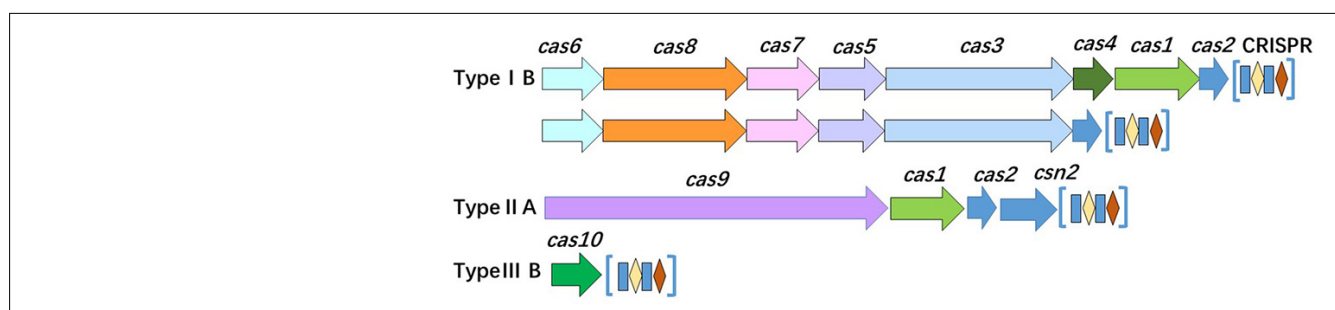

FIGURE 7 | Organization of the CRISPR-Cas system in the isolates. The CRISPR-Cas loci of class 1 (types IB and IIIB) and class 2 (type IIA) are presented.

to support public health (Halbedel et al., 2018; Allard et al., 2019). The cgMLST and SNP have the advantage of high discriminatory power and are widely used for surveillance by appropriate public health analysts and epidemiologists (Hurley et al., 2019; Jagadeesan et al., 2019; Rivas et al., 2019; Papić et al., 2020), whereas CRISPR subtyping is rarely used for Listeria molecular epidemic investigations. In the present study, based on isolates from raw pork meat, WGS was applied to obtain information on cgMLST, SNP, and the CRISPR-Cas system. The same clusters were classified by either cgMLST or SNP. It was observed that isolates from clinical sporadic and outbreak cases could be clustered together with the pork isolates obtained in this study as they had close relationships, which was reflected in the SNP differences. Furthermore, they indicated that pork isolates have a potentially high risk of causing human listeriosis along the food chain.

CRISPR-Cas systems are adaptive immunity systems in many prokaryotic microorganisms; they confer defense capabilities against horizontal gene transfer elements and display variations 
TABLE 2 | Organization of Cas proteins and CRISPRs.

\begin{tabular}{|c|c|c|c|c|}
\hline Types & Cas proteins & STs & Number of Spacers & Isolates \\
\hline & & & $16+157$ & 493 \\
\hline & Cas6, Cas8, Cas7, Cas5, Cas3, Cas4, Cas1 (999 bp), Cas2 & ST155 & $10+58$ & $309,310,311,472$ \\
\hline & & & $10+57$ & 318 \\
\hline & & & $10+61$ & 305 \\
\hline & & & $22+156$ & 465 \\
\hline & & & $22+143$ & 464 \\
\hline & & ST619 & 28 & $471,478,476$ \\
\hline & & & 49 & 477 \\
\hline & & & 78 & 469 \\
\hline & Cas6, Cas8, Cas7, Cas5, Cas3, Cas2 & ST121 & $5+35$ & $306,315,483,312,461,481,467$ \\
\hline & & & 27 & 312 \\
\hline & & & 40 & 461 \\
\hline & & & 44 & 481 \\
\hline & & & 47 & 467 \\
\hline & & & 22 & 320 \\
\hline & & & 21 & 319 \\
\hline \multirow[t]{3}{*}{ Type IIIb } & Cas 10 & ST9 & $4+1$ & $313,297,307,487$ \\
\hline & & & $4+1+1$ & 321 \\
\hline & & & $4+1+1+1+1$ & 459 \\
\hline \multirow[t]{9}{*}{ Cas3 } & Cas3 $^{\#}$ Cas $^{\#}{ }^{\text {Cas3 }}{ }^{\#}$ Cas3 $^{\#}$ & ST8 & $1+7+2+0$ & 316 \\
\hline & $\operatorname{Cas}^{\varphi}{ }^{\varphi} \operatorname{Cas}^{\star}{ }^{*}$ Cas $3^{*} \operatorname{Cas}^{*}$ & ST8 & $1+7+0+0$ & 298 \\
\hline & Cas3 $^{\#}$ Cas3 $^{\#}$ & ST204 & $2+0$ & 482 \\
\hline & Cas3 $^{\#}$ Cas $^{\#}$ & ST204 & $1+0$ & 491 \\
\hline & 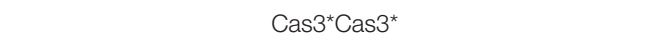 & ST204 & $2+0$ & 495 \\
\hline & $\operatorname{Cas}^{*}{ }^{*} \operatorname{Cas}^{*}$ & ST11 & $7+0$ & 299 \\
\hline & Cas3 $^{\#}$ & ST2O4 & 1 & 314 \\
\hline & Cas3 ${ }^{\#}$ & ST204 & 0 & 494,496 \\
\hline & Cas3 $^{*}$ & ST9, ST204 & 0 & 304,492 \\
\hline
\end{tabular}

"+" means that there are two separate CRISPR loci in the genome.

Cas3*, Cas3 (3,540 bp); Cas3", Cas3 (3,573 bp); Cas3 ${ }^{\varphi}$, Cas3 (1,569 bp).

in different species (Marraffini, 2015; Koonin et al., 2017). CRISPR-based genotyping applications have been exploited for some species, which have been classified into 2 classes, 6 types, and 33 subtypes (Makarova et al., 2020). In the case of Salmonella enterica serovar Typhimurium, the profile of spacer arrangements has strong association with strain phylogeny (Xie et al., 2019). However, its application to L. monocytogenes is limited owing to difficulties in acquiring CRISPR structures from the Listeria genotype, particularly in the lineage I strains (Di et al., 2014). RliB-CRISPR is ubiquitously distributed among
Listeria genome (Sesto et al., 2014). Taylor and Stasiewicz (2019) indicated that CRISPR subtyping cannot improve differentiation of persistent and sporadic L. monocytogenes strains. By combining the organization of Cas proteins and the arrangement of CRISPR loci of isolates, we found that types IB, IIA, and IIIB displayed their unique variations in the Cas proteins and spacers, and there were remarkable features of Cas 3 proteins and CRISPRs in other strains. Importantly, L. monocytogenes strains with closely evolutionary relationship were indistinguishable by SNP subtyping, but they could 
be differentiated adequately by CRISPR subtyping. Our results indicated that combining the organization of Cas proteins and CRISPR loci could be a complementary measure to discriminate genetically close "indistinguishable" strains harboring CRISPR. CRISPR-Cas system plays multiple roles beyond adaptive immunity, i.e., gene regulation and virulence (Faure et al., 2019). Understanding the structure and function of CRISPRCas system contributes to develop useful technology and products to control pathogen, i.e., phages of Listeria offer novel tools for detection, differentiation, CRISPR-Cas-assisted phage engineering, diagnostics, and biocontrol (Hagens and Loessner, 2014; Hupfeld et al., 2018; Meile et al., 2020).

Listeria monocytogenes carrying different virulence factors are highly heterogeneous distributions between CCs and lineages (Tavares et al., 2020). The four known Listeria pathogenicity islands play vital roles in $L$. monocytogenes survival and infection in hosts, which were mainly described for lineage I (LIPI-1, -3, and -4) and sublineage II (LIPI-1, and LIPI-2) (Vázquez-Boland et al., 2001; Cotter et al., 2008; Maury et al., 2016; Yin et al., 2019). Maury et al. (2016) found that CC1, CC6, CC4, and CC2 belonging to Lineage I are the dominant CCs causing listeriosis in Western countries, particularly, CC4 carrying both LIPI-1 and 4 confers hypervirulence by enhancing invasion into the CNS and placenta. Whereas, sporadic listeriosis is the primary feature of clinical cases recorded in China, the CCs -87, -8, -9, -121, -155, and -619 were present in human clinical cases (Li et al., 2019; Zhang et al., 2019a; Yin et al., 2020), CC87 isolates harboring LIPI-1, -3 , and -4 were one of the most prevalent hypervirulent CC in clinical isolates (Li et al., 2019; Zhang et al., 2019a; Yin et al., 2020). In this study, none of CC87 strains were isolated, while a unique clonal complex CC619 isolates carrying LIPI-1, -3, and -4 belonging to lineage II were isolated from different supermarket chains, which have closely evolutionary relationship with clinical ST619 isolates (50 SNP differences), suggesting that they have high risk to cause listeriosis along food production chain. Thus, the CC isolates observed in this study implied that although the occurrence of putatively hypervirulent isolates was not high, all isolates associated with the pork production chain could have substantial public health implications.

In this study, WGS was applied as an investigation tool to detect the status of $L$. monocytogenes contamination via raw pork sold in supermarkets. Specific CRISPR-Cas systems contribute to improve the discrimination of genetically close "indistinguishable" strains. Some supermarket chains have different supply channels which results in the diversity of isolates. Multiple farms supplying pigs to the same slaughterhouse possibly caused the abundant diversity of the strains isolated from same supermarket chains. Importantly, closely evolutionary

\section{REFERENCES}

Alcock, B. P., Raphenya, A. R., Lau, T., Tsang, K. K., Bouchard, M., Edalatmand, A., et al. (2020). CARD 2020: antibiotic resistance surveillance with the comprehensive antibiotic resistance database. Nucleic Acids Res. 48, D517D525. doi: 10.1093/nar/gkz935

Allard, M. W., Strain, E., Rand, H., Melka, D., Correll, W. A., Hintz, L., et al. (2019). Whole genome sequencing uses for foodborne contamination and compliance: discovery of an emerging contamination event in an ice cream facility using relationship exists in isolates from the pork sold in different supermarket chains and supplied from the same slaughterhouse, which indicates that L. monocytogenes spread along the slaughter production chain. The genetic similarities between isolates from different supermarket chains suggest the importance and necessity of tracking and detecting the sources of meat supply at both the slaughterhouse and farm levels. The high isolation rates from contaminated pork meat supplied via 11 supermarket chains in Wuhan city signify the urgent need to strengthen pathogen surveillance systems.

\section{DATA AVAILABILITY STATEMENT}

The data presented in this study are deposited in the European Nucleotide Archive repository, accession number PRJEB41790.

\section{AUTHOR CONTRIBUTIONS}

ML and YW designed the experiments. YW and QJ performed experiments and analyzed the results. SL guided the data analysis. YW and ML wrote the manuscript. All authors read and approved the final manuscript.

\section{FUNDING}

This work was supported by the National Key Research and Development Program of China (2018YFD0500502).

\section{SUPPLEMENTARY MATERIAL}

The Supplementary Material for this article can be found online at: https://www.frontiersin.org/articles/10.3389/fmicb. 2021.620482/full\#supplementary-material

Supplementary Figure 1 | Numerical representation of the 64 isolates. (A) Isolation rate of $L$. monocytogenes from pork meat in 3 districts. (B) Isolation rate of $L$. monocytogenes from pork meat in 11 supermarket chains. (C) Ratio of serotypes among strains isolated in 11 supermarket chains. (D) Distribution of sequence type.

Supplementary Figure 2 | Cluster analysis of CRISPR loci in 64 isolates.

Supplementary Table 1 | Farms for E and G slaughterhouses.

Supplementary Table 2 | Information of clinical isolates.

Supplementary Table $\mathbf{3}$ | SNP analysis of pork isolates, international reference strain and clinical isolates.

whole genome sequencing. Infect. Genet. Evol. 73, 214-220. doi: 10.1016/j. meegid.2019.04.026

Bubert, A., Hein, I., Rauch, M., Lehner, A., Yoon, B., Goebel, W., et al. (1999). Detection and differentiation of Listeria spp. by a single reaction based on multiplex PCR. Appl. Environ. Microbiol. 65, 4688-4692. doi: 10.1128/AEM.65. 10.4688-4692.1999

Chen, L., Yang, J., Yu, J., Yao, Z., Sun, L., Shen, Y., et al. (2005). VFDB: a reference database for bacterial virulence factors. Nucleic Acids Res. 33, D325-D328. doi: $10.1093 /$ nar/gki008 
Chen, M., Cheng, J., Zhang, J., Chen, Y., Zeng, H., Xue, L., et al. (2019). Isolation, potential virulence, and population diversity of Listeria monocytogenes from meat and meat products in china. Front. Microbiol. 10:946. doi: 10.3389/fmicb. 2019.00946

Chen, Y., Gonzalez-Escalona, N., Hammack, T. S., Allard, M. W., Strain, E. A., and Brown, E. W. (2016). Core genome multilocus sequence typing for identification of globally distributed clonal groups and differentiation of outbreak strains of Listeria monocytogenes. Appl. Environ. Microbiol. 82, 62586272. doi: 10.1128/AEM.01532-16

Chenal-Francisque, V., Lopez, J., Cantinelli, T., Caro, V., Tran, C., Leclercq, A., et al. (2011). Worldwide distribution of major clones of Listeria monocytogenes. Emerg. Infect. Dis. 17, 1110-1112. doi: 10.3201/eid/1706.101778

Choi, M. J., Jackson, K. A., Medus, C., Beal, J., Rigdon, C. E., Cloyd, T. C., et al. (2014). Notes from the field: multistate outbreak of listeriosis linked to softripened cheese-United States, 2013. MMWR Morb. Mortal. Wkly Rep. 63, 294-295.

Clausen, P. T. L. C., Aarestrup, F. M., and Lund, O. (2018). Rapid and precise alignment of raw reads against redundant databases with KMA. BMC Bioinformatics. 19:307. doi: 10.1186/s12859-018-2336-6

Cotter, P. D., Draper, L. A., Lawton, E. M., Daly, K. M., Groeger, D. S., Casey, P. G., et al. (2008). Listeriolysin S, a novel peptide haemolysin associated with a subset of lineage I Listeria monocytogenes. PLoS pathog. 4:e1000144. doi: 10.1371/journal.ppat.1000144

de Valk, H., Vaillant, V., Jacquet, C., Rocourt, J., Le Querrec, F., Stainer, F., et al. (2001). Two consecutive nationwide outbreaks of listeriosis in france, october 1999-february 2000. Epidemiol. Rev. 154, 944-950. doi: 10.1093/aje/154. 10.944

Di, H., Ye, L., Yan, H., Meng, H., Yamasak, S., and Shi, L. (2014). Comparative analysis of CRISPR loci in different Listeria monocytogenes lineages. Biochem. Biophys. Res. Commun. 454, 399-403. doi: 10.1016/j.bbrc.2014.10.018

Doumith, M., Buchrieser, C., Glaser, P., Jacquet, C., and Martin, P. (2004). Differentiation of the major Listeria monocytogenes serovars by multiplex PCR. J. Clin. Microbiol. 42, 3819-3822. doi: 10.1128/JCM.42.8.3819-3822.2004

EFSA, and ECDC. (2019). The European Union one health 2018 zoonoses report. EFSA J. 17:e05926. doi: 10.2903/j.efsa.2019.5926

Faure, G., Makarova, K. S., and Koonin, E. V. (2019). CRISPR-cas: complex functional networks and multiple roles beyond adaptive immunity. J. Mol. Biol. 431, 3-20. doi: 10.1016/j.jmb.2018.08.030

Félix, B., Feurer, C., Maillet, A., Guillier, L., Boscher, E., Kerouanton, A., et al. (2018). Population genetic structure of Listeria monocytogenes strains isolated from the pig and pork production chain in france. Front. Microbiol. 9:684. doi: 10.3389/fmicb.2018.00684

Figueroa-López, A. M., Maldonado-Mendoza, I. E., López-Cervantes, J., VerdugoFuentes, A. A., Ruiz-Vega, D. A., Cantú-Soto, E. U. et al. (2019). Prevalence and characterization of Listeria monocytogenes isolated from pork meat and on inert surfaces. Braz. J. Microbiol. 50, 817-824. doi: 10.1007/s42770-019-00073-7

Grissa, I., Vergnaud, G., and Pourcel, C. (2007). CRISPRFinder: a web tool to identify clustered regularly interspaced short palindromic repeats. Nucleic Acids Res. 35, W52-W57. doi: 10.1093/nar/gkm360

Hagens, S., and Loessner, M. J. (2014). Phages of Listeria offer novel tools for diagnostics and biocontrol. Front. Microbiol. 5:159. doi: 10.3389/fmicb.2014. 00159

Halbedel, S., Prager, R., Fuchs, S., Trost, E., Werner, G., Flieger, A. et al. (2018). Whole-Genome Sequencing of recent Listeria monocytogenes isolates from Germany reveals population structure and disease clusters. J. Clin. Microbiol. 56, e118-e119. doi: 10.1128/JCM.00119-18

Halbedel, S., Wilking, H., Holzer, A., Kleta, S., Fischer, M. A., Lüth, S., et al. (2020). Large nationwide outbreak of invasive listeriosis associated with blood sausage, germany, 2018-2019. Emerg. Infect. Dis. 26, 1456-1464. doi: 10.3201/eid2607. 200225

Harakeh, S., Saleh, I., Zouhairi, O., Baydoun, E., Barbour, E., and Alwan, N. (2009). Antimicrobial resistance of Listeria monocytogenes isolated from dairy-based food products. Sci. Total Environ. 407, 4022-4027. doi: 10.1016/j.scitotenv. 2009.04.010

Hasegawa, M., Iwabuchi, E., Yamamoto, S., Esaki, H., Kobayashi, K., Ito, M., et al. (2013). Prevalence and characteristics of Listeria monocytogenes in bovine colostrum in Japan. J. Food Prot. 76, 248-255. doi: 10.4315/0362-028X.JFP$12-278$
Hupfeld, M., Trasanidou, D., Ramazzini, L., Klumpp, J., Loessner, M. J., and Kilcher, S. (2018). A functional type II-A CRISPR-Cas system from Listeria enables efficient genome editing of large non-integrating bacteriophage. Nucleic Acids Res. 46, 6920-6933. doi: 10.1093/nar/gky544

Hurley, D., Luque-Sastre, L., Parker, C. T., Huynh, S., Eshwar, A. K., Nguyen, S. V., et al. (2019). Whole-genome sequencing-based characterization of 100 Listeria monocytogenes isolates collected from food processing environments over a four-year period. $m$ Sphere 4, e219-e252. doi: 10.1128/mSphere.00252-19

Jagadeesan, B., Baert, L., Wiedmann, M., and Orsi, R. H. (2019). Comparative analysis of tools and approaches for source tracking Listeria monocytogenes in a food facility using whole-genome sequence data. Front. Microbiol. 10:947. doi: 10.3389/fmicb.2019.00947

Koonin, E. V., Makarova, K. S., and Zhang, F. (2017). Diversity, classification and evolution of CRISPR-cas systems. Curr. Opin. Microbiol. 37, 67-78. doi: 10.1016/j.mib.2017.05.008

Letunic, I., and Bork, P. (2007). Interactive tree of life (iTOL): an online tool for phylogenetic tree display and annotation. Bioinformatics. 23, 127-128. doi: 10.1093/bioinformatics/btl529

Li, H., and Durbin, R. (2009). Fast and accurate short read alignment with burrowswheeler transform. Bioinformatics 25, 1754-1760. doi: 10.1093/nar/gkm360

Li, W., Bai, L., Fu, P., Han, H., Liu, J., and Guo, Y. (2018). The epidemiology of Listeria monocytogenes in china. Foodborne Pathog. Dis. 15, 459-466. doi: 10.1089/fpd.2017.2409

Li, W., Bai, L., Ma, X., Zhang, X., Li, X., Yang, X., et al. (2019). Sentinel listeriosis surveillance in selected hospitals, china, 2013-2017. Emerg. Infect. Dis. 25, 2274-2277. doi: 10.3201/eid2512.180892

Makarova, K. S., Wolf, Y. I., Iranzo, J., Shmakov, S. A., Alkhnbashi, O. S., Brouns, S., et al. (2020). Evolutionary classification of CRISPR-cas systems: a burst of class 2 and derived variants. Nat. Rev. Microbiol. 18, 67-83. doi: 10.1038/s41579-0190299-x

Marraffini, L. A. (2015). CRISPR-Cas immunity in prokaryotes. Nature. 526, 55-61. doi: $10.1038 /$ nature 15386

Matle, I., Mbatha, K. R., Lentsoane, O., Magwedere, K., Morey, L., and Madoroba, E. (2019). Occurrence, serotypes, and characteristics of Listeria monocytogenes in meat and meat products in south africa between 2014 and 2016. J. Food Safety. 39:e12629. doi: 10.1111/jfs. 12629

Matle, I., Mbatha, K. R., and Madoroba, E. (2020). A review of Listeria monocytogenes from meat and meat products: Epidemiology, virulence factors, antimicrobial resistance and diagnosis. Onderstepoort J. Vet. Res. 87, e1-e20. doi: 10.4102/ojvr.v87il.1869

Maury, M. M., Tsai, Y. H., Charlier, C., Touchon, M., Chenal-Francisque, V., Leclercq, A., et al. (2016). Uncovering Listeria monocytogenes hypervirulence by harnessing its biodiversity. Nat. Genet. 48, 308-313. doi: 10.1038/ ng.3501

McKenna, A., Hanna, M., Banks, E., Sivachenko, A., Cibulskis, K., Kernytsky, A., et al. (2010). The genome analysis toolkit: a mapreduce framework for analyzing next-generation DNA sequencing data. Genome Res. 20, 1297-1303. doi: $10.1101 /$ gr.107524.110

Meile, S., Sarbach, A., Du, J., Schuppler, M., Saez, C., Loessner, M. J., et al. (2020). Engineered reporter phages for rapid bioluminescence-based detection and differentiation of viable Listeria cells. Appl. Environ. Microbiol. 86, e420-e442. doi: 10.1128/AEM.00442-20

Moreno, L. Z., Paixão, R., Gobbi, D. D., Raimundo, D. C., Ferreira, T. P., Moreno, A. M., et al. (2014). Characterization of antibiotic resistance in Listeria spp. isolated from slaughterhouse environments, pork and human infections. J. Infect. Dev. Ctries. 8, 416-423. doi: 10.3855/jidc.4188

Moura, A., Criscuolo, A., Pouseele, H., Maury, M. M., Leclercq, A., Tarr, C., et al. (2016). Whole genome-based population biology and epidemiological surveillance of Listeria monocytogenes. Nat. Microbiol. 2:16185. doi: 10.1038/ nmicrobiol.2016.185

Orsi, R. H., den Bakker, H. C., and Wiedmann, M. (2011). Listeria monocytogenes lineages: genomics, evolution, ecology, and phenotypic characteristics. Int. J. Med. Microbiol. 301, 79-96. doi: 10.1016/j.ijmm.2010.05.002

Papić, B., Kušar, D., Zdovc, I., Golob, M., and Pate, M. (2020). Retrospective investigation of listeriosis outbreaks in small ruminants using different analytical approaches for whole genome sequencing-based typing of Listeria monocytogenes. Infect. Genet. Evol. 77:104047. doi: 10.1016/j.meegid.2019. 104047 
Ragon, M., Wirth, T., Hollandt, F., Lavenir, R., Lecuit, M., Le Monnier, A., et al. (2008). A new perspective on Listeria monocytogenes evolution. PLoS Pathog. 4:e1000146. doi: 10.1371/journal.ppat.1000146

Rivas, L., Dupont, P. Y., Wilson, M., Rohleder, M., and Gilpin, B. (2019). An outbreak of multiple genotypes of Listeria monocytogenes in new zealand linked to contaminated ready-to-eat meats - a retrospective analysis using whole-genome sequencing. Lett. Appl. Microbiol. 69, 392-398. doi: 10.1111/lam. 13227

Salcedo, C., Arreaza, L., Alcalá, B., de la Fuente, L., and Vázquez, J. A. (2003). Development of a multilocus sequence typing method for analysis of Listeria monocytogenes clones. J. Clin. Microbiol. 41, 757-762. doi: 10.1128/jcm.41.2. 757-762.2003

Scortti, M., Han, L., Alvarez, S., Leclercq, A., Moura, A., Lecuit, M., et al. (2018). Epistatic control of intrinsic resistance by virulence genes in Listeria. PLoS Genet. 14:e1007525. doi: 10.1371/journal.pgen.1007525

Scortti, M., Lacharme-Lora, L., Wagner, M., Chico-Calero, I., Losito, P., and Vázquez-Boland, J. A. (2006). Coexpression of virulence and fosfomycin susceptibility in Listeria: molecular basis of an antimicrobial in vitro-in vivo paradox. Nat. Med. 12, 515-517. doi: 10.1038/nm1396

Sesto, N., Touchon, M., Andrade, J. M., Kondo, J., Rocha, E. P. C., Arraiano, C. M., et al. (2014). A PNPase dependent CRISPR System in Listeria. PLoS Genet. 10:e1004065. doi: 10.1371/journal.pgen.1004065

Smith, A., Hearn, J., Taylor, C., Wheelhouse, N., Kaczmarek, M., Moorhouse, E., et al. (2019). Listeria monocytogenes isolates from ready to eat plant produce are diverse and have virulence potential. Int. J. Food Microbiol. 299, 23-32. doi: 10.1016/j.ijfoodmicro.2019.03.013

Tavares, R. M., Silva, D. A. L. D., Camargo, A. C., Yamatogi, R. S., and Nero, L. A. (2020). Interference of the acid stress on the expression of llsX by Listeria monocytogenes pathogenic island 3 (LIPI-3) variants. Food Res. Int. 132:109063. doi: $10.1016 /$ j.foodres.2020.109063

Taylor, A. J., and Stasiewicz, M. J. (2019). CRISPR-Based Subtyping using whole genome sequence data does not improve differentiation of persistent and sporadic Listeria monocytogenes strains. J. Food Sci. 84, 319-326. doi: 10.1111/ 1750-3841.14426

Thomas, J., Govender, N., McCarthy, K. M., Erasmus, L. K., Doyle, T. J., Allam, M., et al. (2020). Outbreak of listeriosis in south africa associated with processed meat. N. Engl. J. Med. 382, 632-643. doi: 10.1056/ NEJMoa1907462

Vázquez-Boland, J. A., Kuhn, M., Berche, P., Chakraborty, T., Domínguez-Bernal, G., Goebel, W., et al. (2001). Listeria pathogenesis and molecular virulence determinants. Clin. Microbiol. Rev. 14, 584-640. doi: 10.1128/CMR.14.3.584640.2001
Wang, Y., Luo, L., Li, Q., Wang, H., Wang, Y., Sun, H., et al. (2019). Genomic dissection of the most prevalent Listeria monocytogenes clone, sequence type ST87, in China. BMC Genom. 20:1014. doi: 10.1186/s12864-019-6399-1

Xie, X., Wang, Z., Zhang, K., Li, Y., Hu, Y., Pan, Z., et al. (2019). Pig as a reservoir of CRISPR type TST4 Salmonella enterica serovar Typhimurium monophasic variant during 2009-2017 in China. Emerg. Microb. Infect. 9, 1-4. doi: 10.1080/ 22221751.2019.1699450

Yin, Y., Doijad, S., Wang, W., Lian, K., Pan, X., Koryciński, I., et al. (2020). Genetic Diversity of Listeria monocytogenes isolates from invasive listeriosis in china. Foodborne Pathog. Dis. 17, 215-227. doi: 10.1089/fpd.2019.2693

Yin, Y., Tan, W., Wang, G., Kong, S., Zhou, X., Zhao, D., et al. (2015). Geographical and longitudinal analysis of Listeria monocytogenes genetic diversity reveals its correlation with virulence and unique evolution. Microbiol. Res. 175, 84-92. doi: 10.1016/j.micres.2015.04.002

Yin, Y., Yao, H., Doijad, S., Kong, S., Shen, Y., Cai, X., et al. (2019). A hybrid sub-lineage of Listeria monocytogenes comprising hypervirulent isolates. Nat. Commun. 10:4283. doi: 10.1038/s41467-019-12072-1

Zankari, E., Hasman, H., Cosentino, S., Vestergaard, M., Rasmussen, S., Lund, O., et al. (2012). Identification of acquired antimicrobial resistance genes. J. Antimicrob. Chemother. 67, 2640-2644. doi: 10.1093/jac/dks261

Zhang, X., Niu, Y., Liu, Y., Lu, Z., Wang, D., Cui, X., et al. (2019a). Isolation and characterization of clinical Listeria monocytogenes in beijing, china, 2014-2016. Front. Microbiol. 10:981. doi: 10.3389/fmicb.2019.00981

Zhang, Y., Dong, S., Chen, H., Chen, J., Zhang, J., Zhang, Z., et al. (2019b). Prevalence, genotypic characteristics and antibiotic resistance of Listeria monocytogenes from retail foods in bulk in Zhejiang province, China. Front. Microbiol. 10:1710. doi: 10.3389/fmicb.2019.01710

Zuber, I., Lakicevic, B., Pietzka, A., Milanov, D., Djordjevic, V., Karabasil, N., et al. (2019). Molecular characterization of Listeria monocytogenes isolates from a small-scale meat processor in montenegro, 2011-2014. Food Microbiol. 79, 116-122. doi: 10.1016/j.fm.2018.12.005

Conflict of Interest: The authors declare that the research was conducted in the absence of any commercial or financial relationships that could be construed as a potential conflict of interest.

Copyright (c) 2021 Wang, Ji, Li and Liu. This is an open-access article distributed under the terms of the Creative Commons Attribution License (CC BY). The use, distribution or reproduction in other forums is permitted, provided the original author(s) and the copyright owner(s) are credited and that the original publication in this journal is cited, in accordance with accepted academic practice. No use, distribution or reproduction is permitted which does not comply with these terms. 